
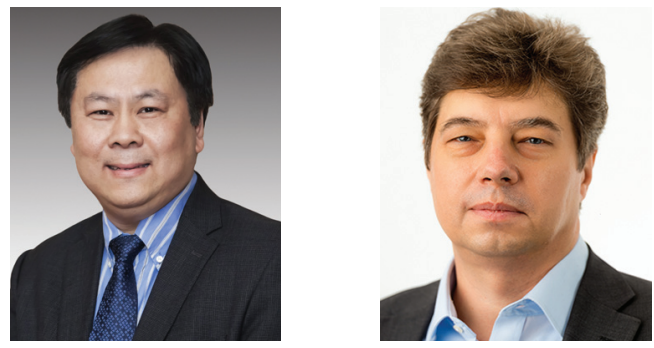

\title{
Laser: sixty years of advancement
}

In 1953, microwave amplification by stimulated emission of radiation (maser) was demonstrated. It took another seven painstaking years to achieve light amplification by stimulated emission of radiation (laser), and the world has changed. Without laser, not only would this journal not exist, but many experiences which we take for granted in everyday life-from supermarket checkout to downloading movieswould not be here. The impact of lasers on each and every research field cannot be exaggerated. This is evidenced by the impressive number of Nobel Prizes awarded for the development or use of lasers. ${ }^{1}$ From imaging of nanoscale features of biological cells and observing chemical reactions in real time to detecting gravitational waves and propelling satellites to distant galaxies, lasers are there for us. Lasers come in an assortment of colors, shapes, and sizes. The development of ever evolving laser systems continues, and we see many new advances and applications almost every day.

We are proud that Advanced Photonics was able to report some of the most striking recent advances in laser science and new applications. This includes both traditional lasers ${ }^{2}$ and nanoscale lasers, ${ }^{3}$ lasers generating two different beams at once ${ }^{4}$ and lasers generating combs of wavelengths, ${ }^{5}$ novel fibre lasers ${ }^{6-9}$ and soliton lasers $;,{ }^{5,8,10}$ we have them all for you. While nearly all other papers published in this journal make use of lasers, some of the mind-boggling optical techniques such as ultrafast photography ${ }^{11-13}$ and ultrahigh-resolution spectroscopy ${ }^{14}$ would not be possible at all without lasers.

For this issue, we commissioned two review articles to see what future holds for the laser science. One looks at newly discovered laser applications of wonder materials - perovskites - capable of providing emission from near-ultraviolet to near-infrared wavelengths. ${ }^{15}$ The other discusses lasers of such a small size that they should not exist in traditional laser physics. ${ }^{16}$ These nanolasers have opened up a new chapter in laser science: surface plasmon amplification by stimulated emission of radiation (spaser) with myriad new applications in ultrasensing, biology and high-density on-chip communications.

Over two years since its inception, Advanced Photonics has covered the new trends in lasers faithfully, and we will continue to report many new advancements in lasers and laser applications in the years to come.

\section{References}

1. J. Dudley, "Light, lasers, and the Nobel Prize," Adv. Photonics 2(5), 050501 (2020).
2. H. Linnenbank et al., "Robust and rapidly tunable light source for SRS/CARS microscopy with low-intensity noise," Adv. Photonics 1(5), 055001 (2019).

3. C.-Z. Ning, "Semiconductor nanolasers and the size-energyefficiency challenge: a review," Adv. Photonics 1(1), 014002 (2019).

4. J. Fan et al., "Two-channel, dual-beam-mode, wavelength-tunable femtosecond optical parametric oscillator," Adv. Photonics 2(4), 045001 (2020).

5. W. Wang, L. Wang, and W. Zhang, "Advances in soliton microcomb generation," Adv. Photonics 2(3), 034001 (2020).

6. Y. Song et al., "Recent progress on optical rogue waves in fiber lasers: status, challenges, and perspectives," Adv. Photonics 2(2), 024001 (2020).

7. U. Tegin et al., "Single-mode output by controlling the spatiotemporal nonlinearities in mode-locked femtosecond multimode fiber lasers," Adv. Photonics 2(5), 056005 (2020).

8. Z. Qin et al., "Mode-locked 2.8- $\mu \mathrm{m}$ fluoride fiber laser: from soliton to breathing pulse," Adv. Photonics 1(6), 065001 (2019).

9. Z. Ding et al., "All-fiber ultrafast laser generating gigahertz-rate pulses based on a hybrid plasmonic microfiber resonator," $A d v$. Photonics 2(2), 026002 (2020).

10. X. Liu and Y. Cui, "Revealing the behavior of soliton buildup in a mode-locked laser," Adv. Photonics 1(1), 016003 (2019).

11. D. Qi et al., "Single-shot compressed ultrafast photography: a review," Adv. Photonics 2(1), 014003 (2020).

12. X. Zeng et al., "High-spatial-resolution ultrafast framing imaging at 15 trillion frames per second by optical parametric amplification," Adv. Photonics 2(5), 056002 (2020).

13. K. Lin et al., "Spatiotemporal rotational dynamics of laser-driven molecules," Adv. Photonics 2(2), 024002 (2020).

14. J. Chen et al., "Adaptive-sampling near-Doppler-limited terahertz dual-comb spectroscopy with a free-running single-cavity fiber laser," Adv. Photonics 2(3), 036004 (2020).

15. Z. Cao et al., "Optical studies of semiconductor perovskite nanocrystals for classical optoelectronic applications and quantum information technologies: a review," Adv. Photonics 2(5), 054001 (2020).

16. M. I. Stockman, "Brief history of spaser from conception to the future," Adv. Photonics 2(5), 054002 (2020).

Xiao-Cong (Larry) Yuan Co-Editor-in-Chief

Anatoly Zayats Co-Editor-in-Chief

(C) The Authors. Published by SPIE and CLP under a Creative Commons Attribution 4.0 Unported License. Distribution or reproduction of this work in whole or in part requires full attribution of the original publication, including its DOI. [DOI: 10.1117/1 .AP.2.5.050101] 